\title{
Predicting fractures using trabecular patterns on panoramic radiographs
}

\author{
Wil Geraets ${ }^{1}$ - Grethe Jonasson ${ }^{2,3}$ • Magnus Hakeberg ${ }^{3}$
}

Received: 13 December 2016 / Accepted: 5 May 2017 /Published online: 1 June 2017

(C) The Author(s) 2017. This article is an open access publication

\begin{abstract}
Objectives The observer score of the trabecular pattern on panoramic radiographs is known to be a strong predictor of bone fractures. The aim of this study was to enhance the predictive power of panoramic radiographs by means of texture analysis methods.

Material and methods The study followed 304 postmenopausal women during 26 years. At the beginning of the study, panoramic radiographs were obtained. One observer assessed the trabecular pattern in the premolar region as dense, sparse, or alternating dense and sparse. In addition, on each radiograph, a region of interest was selected in the molar/ premolar region and analyzed with texture analysis procedures. During 26 years of follow-up, 115 women suffered a fracture of the hip, spine, leg, or arm. Logistic regression was applied to test the predictive power of various variables with respect to fractures.

Results Of all variables, the observer score of the trabecular pattern correlated strongest with the occurrence of fractures. By itself, the score yielded an ROC curve with an area of 0.80 under the curve. Combining the observer score with the texture analysis features increased the area under the ROC curve to 0.85 .
\end{abstract}

Wil Geraets

w.geraets@acta.nl

1 Department of Oral and Maxillofacial Radiology, Academic Centre for Dentistry Amsterdam (ACTA), Gustav Mahlerlaan 3004, 1081 LA Amsterdam, The Netherlands

2 Research \& Development Unit in Southern Älvsborg County, Sven Eriksonplatsen 4, 50338 Borås, Sweden

3 Department of Behavioral and Community Dentistry, Institute of Odontology, University of Gothenburg, Medicinaregatan 12E, Gothenburg, Sweden
Conclusions The trabecular pattern on panoramic radiographs provides a strong predictor of fractures, at least for postmenopausal women. The assessment by an observer combined with texture analysis procedures yields a predictive power that parallels best known predictions in literature.

Clinical relevance This study illustrates that panoramic radiographs are state of the art predictors of postcranial fractures.

Keywords Fracture prediction - Panoramic radiographs . Trabecular pattern $\cdot$ Observer score $\cdot$ ROC analysis

\section{Introduction}

The greatest complication of bone disease, especially osteoporosis, is the occurrence of fractures. Some $40 \%$ of Caucasian women aged 50 years or more experience a fracture of the hip, spine, or wrist during their life. Fractures of the wrist are the most common, but fractures of the hip are the most serious in terms of mortality, morbidity, and cost $[1,2]$.

The lifetime risk for a hip fracture lies between 14 and 23\% among Caucasian women in Europe and the USA and is likely to increase as mortality for other conditions declines $[1,3]$. Worldwide, there is substantial variation in hip fracture incidence between populations. Even in Europe, the risk for hip fracture varies about three-fold between countries [3].

The WHO defined osteoporosis as a systemic skeletal disease characterized by reduced bone mineral density (BMD) and microarchitectural deterioration of bone tissue leading to increased risk of fractures. The organization recommended to use BMD measurements to diagnose osteoporosis [4]. The prediction of osteoporosis and the prediction of fractures are related subjects, but different nevertheless. The risk of fractures is high when BMD is low, but it is by no means negligible when BMD is normal [5]. Therefore, the majority of 
fractures occur in non-osteoporotic subjects, and BMD measurements are not recommended for population screening [5-9]. Other risk factors for fractures include age, previous fractures, body weight, and body mass index (BMI) [5, 9-11]. A fracture of the hip or spine more than doubles the risk of a subsequent fracture [5, 11].

Fracture prediction uses statistical models to identify people at high risk of fractures. The most common way of measuring the discriminative power of a prediction model is plotting a receiver operating characteristic (ROC) curve [12, 13]. The predictive power of the model then is given by the area under the curve (AUC) [13]. The WHO developed a Fracture Risk Assessment tool (FRAX), to assess the fracture risk based on the most relevant risk factors such as age, sex, weight, height, previous fracture, parent hip fracture, current smoking, glucocorticoid use, rheumatoid arthritis, alcohol use, and femoral neck BMD [9]. With respect to the prediction of major osteoporotic fractures, AUC values up to 0.69 have been reported for FRAX [14, 15]. Alternative tools such as the Osteoporosis Self assessment Tool (OST), the Simple Calculated Osteoporosis Risk Estimation (SCORE), and the FRACTURE Index were developed. OST is based entirely on gender, age, and weight whereas SCORE also involves race, rheumatoid arthritis, estrogen therapy, and fracture history. The FRACTURE Index involves gender, age, weight, BMD, fracture history, and maternal fracture history. Most of these tools performed as accurately as FRAX [16-19]. However, some outperformed FRAX with an AUC value of 0.76 or 0.77 [18-20]. An Australian study reported an impressive AUC of 0.84 using the Garvan algorithm which is based on sex, age, BMD, fall history, and fracture history [21]. Considering that an AUC of 1.00 represents perfect prediction, it seems that at the present state of the art, there is still room for improvement. However, since an element of chance is involved in the occurrence of fractures, perfect prediction is not attainable and it remains to be seen how much the prediction of osteoporotic fractures can be improved.

Moreover, fracture prediction proves to be an elusive issue. An elaborate Norwegian validation study of the Garvan algorithm gave an AUC of only 0.62 [22]. In 2014, an alarming report on fracture prediction was published [23]. The risk of a major osteoporotic fracture was estimated using FRAX, OST, and SCORE, without involving BMD measurements. None of the strategies were substantially better than chance. It was concluded that fracture prediction requires risk factors not included in the current strategies. Therefore, the search for reliable prediction tools should continue [2].

Dental radiographs are among the most frequently made radiographs. Many studies of intraoral and panoramic radiographs report significant relationships with BMD and osteoporosis [24-31]. Studies with respect to the prediction of fractures are less abundant [32-36].
Lindh et al. developed an index to assess the trabecular pattern in periapical radiographs. Validated reference images from mandibular sections with characteristic trabecular patterns and typical distributions of trabecular bone were selected. With help of these reference images, observers assessed the trabecular pattern as dense trabeculation, alternating dense and sparse trabeculation, and sparse trabeculation [37]. The index was adapted by Lindh et al. and by Jonasson et al. [29, 33]. The assessment will be referred to as "observer score."

The observer score of panoramic radiographs was used to predict postcranial fractures. For a group of 518 women, it was found that subjects with dense trabecular patterns had a hazard ratio of 0.07 for fractures in the following 26 years, whereas for subjects with sparse trabecular patterns, the hazard ratio was 3.63 [34]. The present study investigates if further improvement of fracture prediction can be obtained by texture analysis. Various texture analysis methods had been developed by Geraets and co-workers and by White and coworkers [10, 30, 31, 36, 38-44]. After selection of a region of interest (ROI) by an observer, these methods were applied automatically.

The main aim of the study was to maximize the predictive power of the trabecular pattern on panoramic radiographs. The secondary aim of the study was to parallel or even outperform the observer score with texture analysis methods.

\section{Materials and methods}

\section{Subjects}

The present study is based upon the Prospective Population Study of Women in Gothenburg, Sweden, a longitudinal study of perimenopausal women that had been randomly selected from the Revenue Office register. Participants gave their informed consent in accordance with the Helsinki Declaration. The study was approved by the Regional Ethical Review Board in Gothenburg (T453-04 and T075-09). Various medical and dental examinations were performed between 1968 and 2006 [34].

All women who had participated in the first part of the study were invited to also enter into the second part that started in 1980 . Out of them, $73 \%$ underwent the medical and dental reexaminations. At the 1992 follow-up, an extensive nonparticipation analysis was performed. Non-participants were interviewed by means of a telephone call or a letter, and additional information was obtained from national registers and inpatient and outpatient records. Non-participants did not differ significantly from the participants except in long-term survival which was lower among the non-participants [45].

The present study focuses on women born in 1930 and 1922. In 1980, they were 50 or 58 years of age when a panoramic radiograph was made to assess the number of teeth, 
endodontic treatment, and the distance from the cementoenamel junction to the bone crest. In 1992, the survivor participation rate was $69 \%$ for the medical examination and $64 \%$ for the dental examination [46]. At the end of the study in 2006 , the subjects were 76 or 84 years of age. Then, the National Swedish Death Register was used to ascertain whether they were still alive. From the participating survivors, 304 women were selected randomly $(N=170$ and $N=134$ for age 76 and 84, respectively).

The occurrence of fractures between 1980 and 2006 was hospital-verified using the County Patient Register. No fractures of fingers and toes were recorded. Only clinical spine fractures were included. No attempt was made to separate fragility fractures from other fractures [34]. Women who sustained more than one fracture were included only once.

\section{Observer score and clinical variables}

Panoramic radiographs had been obtained during the 1980 examination with a Scanora (Orion Soredex, Helsinki, Finland) with 66-70 kV and $20 \mathrm{~mA}$. In the present study, these radiographs were used to assess the trabecular pattern. They were placed on a light-box in a darkened room, and magnifying lenses $(\times 2)$ were used. One observer (GJ), experienced in classifying the trabecular pattern in oral radiographs, closely inspected the trabecular pattern on the right side of the mandible between the canine and the first molar, at least $2 \mathrm{~mm}$ below the bone crest and at least $2 \mathrm{~mm}$ above the apices of the premolars (Fig. 1). Three radiographs were selected with characteristic trabecular patterns. A dense trabecular pattern has many well-mineralized trabeculae and small intertrabecular spaces. A sparse trabecular pattern has less trabeculae which are less-mineralized, and the intertrabecular spaces are mostly large. An alternating dense and sparse trabecular pattern is dense cervically and sparse apically. Densitometric measurements were performed to validate the reference radiographs [34].

With help of these reference radiographs, the radiographs were classified as dense, sparse, or alternating dense and sparse. In case of uncertainty, the category alternating dense

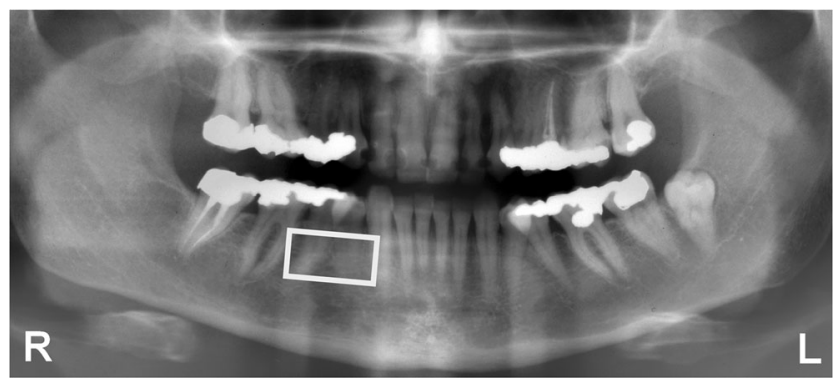

Fig. 1 On the right side of the mandible, the area between the canine and the first molar was assessed as "sparse," "sparse/dense," or "dense" by one observer and sparse was chosen. Crestal bone around teeth with marginal bone loss due to periodontitis was disregarded, as well as sclerotic bone around apices of problematic teeth. The assessment was blinded for fracture status.

A test-retest evaluation was done by the observer who had done the assessment described above and two other observers: an oral and maxillofacial radiologist and a general practitioner. They classified 30 panoramics twice 4 weeks apart [34].

In addition, the clinical variables age, weight, height, and BMI were recorded.

\section{Texture analysis}

The radiographs were scanned with a flatbed scanner (Microtek Medi-2200 plus) at a resolution of 236 pixels per centimeter (600 dpi). First, an observer (WG) manually selected an ROI near the first molar and second premolar on the right side of the mandible. Afterwards, the ROI was adjusted automatically to a fixed size of $650 \times 650$ pixels, corresponding with $2.75 \mathrm{~cm} \times 2.75 \mathrm{~cm}$ (Fig. 2).

The ROIs were subjected to automatic texture analysis procedures measuring various features that had proven their relevance for bone structure and osteoporosis [10, 25, 28, 30, 36, 39, 40, 42-44, 47-49].

First, brightness and contrast were determined. Then, a $3 \times 3$ median filter adjusted isolated pixels with deviating gray values. Next, an unsharp self-masking filter removed largescale variations in gray value, caused by varying thickness of cortex and soft tissues (Fig. 3a). The sample was segmented into a binary image consisting of black and white segments (Fig. 3b).

The binary image was used to measure the number density of black and white segments, as well as their average area, perimeter, and width. The orientation of the binary image was measured in 12 directions $\left(\mathrm{LFD}_{0^{\circ}}, \mathrm{LFD}_{15^{\circ}}, \ldots, \mathrm{LFD}_{165^{\circ}}\right)$ (Fig. 3c) [48]. Next, the white segments were eroded to quantify the average number of nodes and endpoints and the average strut length (Fig. 3d). Finally, the black segments were eroded and quantified in a similar way. The resulting measurements will be referred to as the "image features."

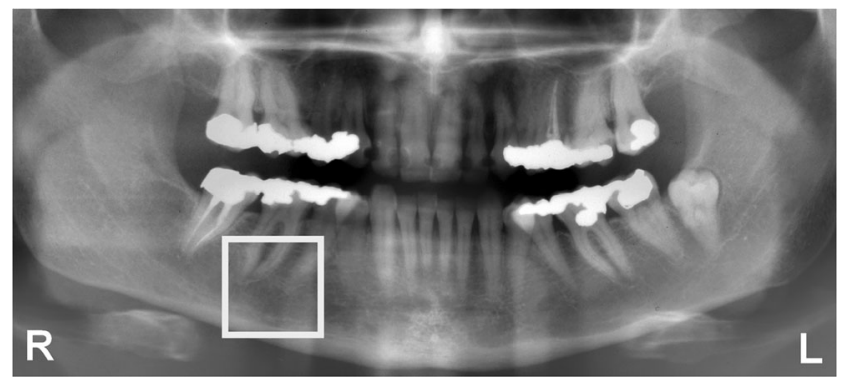

Fig. 2 On the right side of the mandible, an ROI near the premolars was selected. The ROI measured $650 \times 650$ pixels and overlapped the area in Fig. 1 


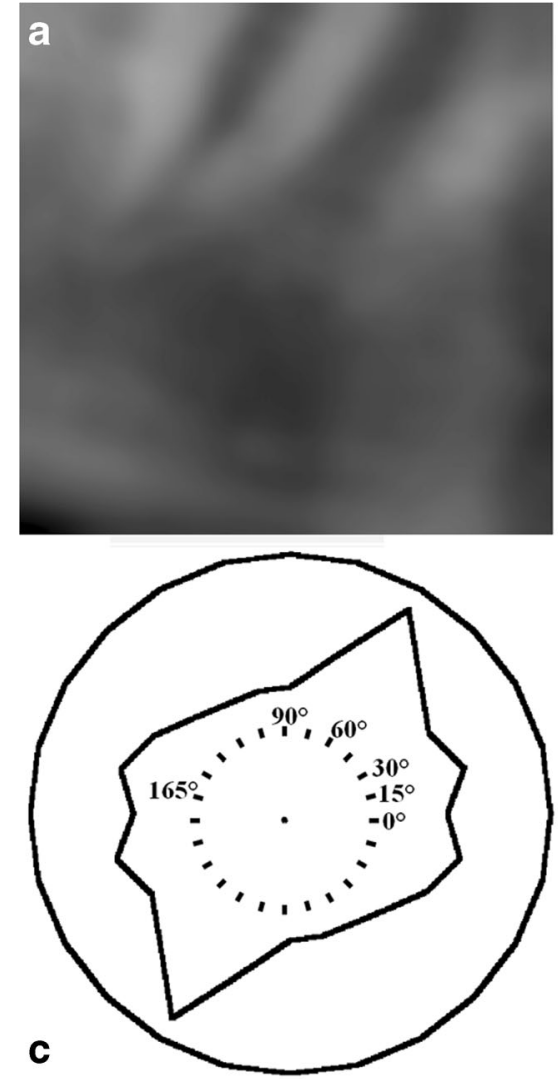

Fig. 3 The ROI in Fig. 2 was subjected to texture analysis. a Blurred version of the ROI. To facilitate segmentation, the blurred version was subtracted from the original shown in Fig. 2. b Segmented ROI consisting of $650 \times 650$ pixels that are black if they belong to intertrabecular spaces, or white if they belong to trabeculae. c LFD orientation of the segmented ROI shown in b. Measurements were made along $0^{\circ}, 15^{\circ}, \ldots$ and $165^{\circ}$.

\section{Statistics}

Cohen's Kappa-statistic was used to calculate the intra- and inter-observer agreement. This statistic was calculated with SAS version 9.2 (SAS Institute Inc., Cary, NC) [34]. Other statistical calculations were done with the SPSS package (version 21; SPSS Inc., Chicago, IL). To define significance, $\alpha=0.05$ was used.

Fracture patients were compared with patients that participated up to the end of the study and remained fracture free. $t$ tests were applied to compare the two groups with respect to age, weight, height, and BMI.

The predictive power of three sets of variables was determined. The first set included the observer score and clinical variables age, weight, height, and BMI. The second set consisted of the image features that had been measured by the texture analysis procedures. The third set of variables combined the first two sets in order to test for any synergetic effect.

Forward stepwise logistic regression was applied to predict the occurrence of fractures. This analysis started with a prediction based on the prevalence of fracture patients and

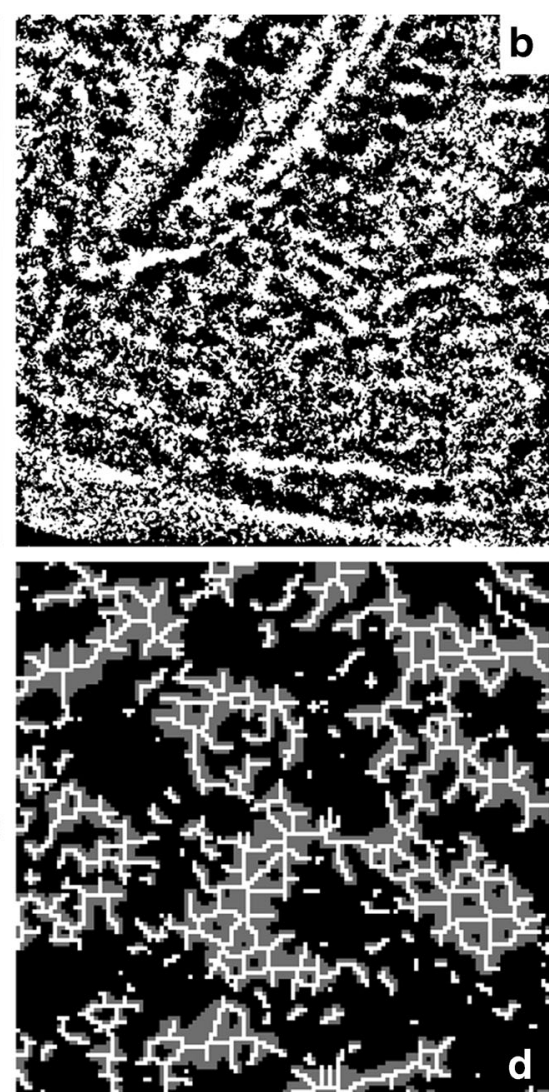

Along opposite directions, the LFD orientation is identical. This particular ROI has maximum LFD orientation along $60^{\circ}$. d Eroded ROI. To illustrate struts, nodes, and endpoints more clearly, the central part of $130 \times 130$ pixels has been taken and magnified 5 times. Eroded trabeculae are shown in gray. Struts are shown in white. Nodes have multiple white neighboring pixels, whereas endpoints have only one

fracture free patients. Then, the variable was added that improved the prediction model most; this was repeated as long as a significant improvement was obtained. After completion of the prediction model, the corresponding ROC curve was constructed.

\section{Results}

Of the 304 women, 115 sustained a fracture between 1980 and 2006. Fractures of the lower arm or wrist were most common (Table 1).

One observer (GJ) assessed the trabecular patterns on the radiographs. Of the 304 subjects, 49 were classified as "dense," 146 as "alternating dense and sparse," and 109 as "sparse" (Table 2). For subjects with a sparse trabecular pattern, the odds ratio of sustaining a fracture was $11.6(95 \%$ confidence interval (CI) 6.7-20.3). And if the pattern had been assessed as dense, the odds ratio of staying free from fractures was 38.8 (CI 5.3-285.5). 
Table 1 Overview of fractures during 26 years of follow-up

304 women in total: 189 without fracture, 115 with fracture

$8 \%$ had 1st fracture between 1980 and 1984

10\% had 1st fracture between 1985 and 1989

15\% had 1st fracture between 1990 and 1994

27\% had 1st fracture between 1995 and 1999

23\% had 1st fracture between 2000 and 2004

$17 \%$ had 1st fracture between 2005 and 2006

$35 \%$ lower arm or wrist

$20 \%$ upper leg or hip

$15 \%$ spine

$12 \%$ upper arm

$10 \%$ lower leg

$9 \%$ other

The Kappa value for the intra-observer agreement of observer GJ was 0.92. The Kappa values for the inter-observer agreement of observer GJ with the two other observers were 0.84 and 0.73 , respectively, which indicates good agreement [34].

The descriptive statistics of age, weight, height, and BMI are provided in Table 3. Height was the only variable that differed for fracture patients and fracture free patients $(p<0.046)$. However, when taking into account that 4 variables were compared simultaneously, it was concluded that there was no significant difference between the two groups.

Logistic regression was applied on the observer score combined with age, weight, height, and BMI. The observer score was selected as the only predictor. This variable yielded an ROC curve with an AUC of 0.800. Age, weight, height, and BMI did not improve the prediction of fractures significantly (Table 4).

Then, logistic regression was applied on the image features resulting in an ROC curve with AUC of 0.603 using the image feature LFD45 ${ }^{\circ}$.

Finally, logistic regression was applied on the image features combined with observer score, age, weight, height, and BMI. The AUC was 0.852 using the observer score, LFD45 and the contrast in the unfiltered ROI.

\section{Discussion}

It is encouraging that the prediction based on the radiographic trabecular pattern yields an ROC curve with AUC 0.85 similar

Table 2 Overview of observer scores of trabecular patterns

\begin{tabular}{crrrr}
\hline & Dense & Alternating dense and sparse & Sparse & Total \\
\hline Fractured & 1 & 35 & 79 & 115 \\
Fracture free & 48 & 111 & 30 & 189 \\
Total & 49 & 146 & 109 & 304 \\
\hline
\end{tabular}

Table 3 Overview of clinical variables (mean $\pm \mathrm{SD}$ ): age in years, weight in $\mathrm{kg}$, height in $\mathrm{cm}, \mathrm{BMI}$ in $\mathrm{kg} / \mathrm{m}^{2}$

\begin{tabular}{cllll}
\hline & Age in 1980 & Weight & Height & BMI \\
\hline Fractured & $54.3 \pm 4.1$ & $65.8 \pm 9.9$ & $164.4 \pm 6.1$ & $24.3 \pm 3.4$ \\
Fracture free & $53.9 \pm 4.0$ & $66.7 \pm 11.0$ & $163.0 \pm 5.8$ & $25.1 \pm 3.7$ \\
$\quad$ Total & $54.1 \pm 4.0$ & $66.3 \pm 10.6$ & $163.5 \pm 5.9$ & $24.8 \pm 3.6$ \\
\hline
\end{tabular}

to the best prediction with AUC 0.84 using the Garvan algorithm [21]. The logistic regression analysis consistently selected the observer score as the most important predictor. If a sparse trabecular pattern was used as a predictor for fractures, then the prediction had a specificity of $84 \%$ and a sensitivity of $69 \%$. These values define a point within distance 0.02 of the ROC curve described by Sandhu and co-workers [21]. Clearly, the observer score by itself can predict osteoporotic fractures nearly as accurately as the best prediction described in literature. Although the present study and the study by Sandhu et al. both are retrospective, they differ in the length of the follow-up, the age distribution, and the nationality of the populations. Therefore, the AUC values should be interpreted with caution.

The present study included only subjects that participated until the end of the study. Since non-participators tended to be less healthy than survivors, it is plausible that fractures and sparse trabecular patterns were more common among nonparticipators. So, any selection bias would probably not favor the prediction of fractures $[50,51]$. Moreover, some of the fractures in this study may not have been fragility fractures. But there is evidence that the association with osteoporosis is similar for high- and low-trauma fractures [11].

A weak point of the present study is that the trabecular pattern was assessed by one observer (GJ) only. To some extent, that is compensated by the good agreement between the observer and two others. That makes it plausible that other observers would have assessed the trabecular patterns similarly. In addition, it is desirable to perform similar studies on other populations since the performance of fracture predictor tools varies over populations.

A strong point of the present study is the long fracture follow-up. The huge investment of time and effort needed for such studies explains why they are rare. Mostly, time is saved by predicting BMD values rather than the occurrence of

Table 4 AUC for prediction of fractures

\begin{tabular}{llll}
\hline & AUC & $95 \%$ CI & Predictors \\
\hline $\begin{array}{l}\text { Observer score and } \\
\text { clinical variables }\end{array}$ & 0.800 & $0.749-0.851$ & Observer score \\
$\begin{array}{l}\text { Image features } \\
\text { Combined }\end{array}$ & 0.603 & $0.537-0.669$ & LFD45 \\
& 0.852 & $0.808-0.894$ & Observer score, \\
& & & LFD45 $5^{\circ}$ contrast \\
\hline
\end{tabular}


fractures. However, since BMD values have low sensitivity for fracture prediction, they cannot replace long-term followup studies entirely.

In literature, there is renewed interest in cortical bone with respect to BMD and fragility [52-54]. Calciolari et al. show that the mandibular cortical width, the panoramic mandibular index, and the Klemetti index are overall useful panoramic measures to screen for low BMD. Yet, the relevance of such measures for postcranial fractures remains to be quantified. Zebaze et al. argue that the relevance of cortical bone with respect to bone fragility has been neglected. They show that in the aging distal radius, the amount of cortical bone loss doubles the amount of trabecular bone loss [54]. Moreover, they show that in the aging femur, the remodeling surface in cortical bone exceeds the remodeling area in trabecular bone. At the age of 29, the pores in the cortex are small spheres of about $0.07 \mathrm{~mm}$ diameter, evenly distributed throughout the cortex. Such pores in the mandibular cortex would be invisible on panoramic radiographs since even the best panoramic devices resolve details of $0.1 \mathrm{~mm}$ minimum. However, at the age of 67 , the pores have increased in size up to $0.5 \mathrm{~mm}$ and their shape is irregular. At the age of 90 , most of the femoral cortex has been trabecularized. If the mandibular cortex degrades in a similar way, then it is plausible that the cortex contributes to the radiographic trabecular pattern. For intraoral radiographs, this holds even more since they resolve smaller details throughout the mandibular bone. The phenomenon that cortical bone gradually becomes trabecularized implies that any technique to distinguish between cortical and trabecular bone can be questioned. It should be realized that the trabecular pattern not necessarily originates from trabecular bone only.

Panoramic radiographs have various geometric distorsions and provide less details than intraoral radiographs. Proper positioning of the patient is necessary to obtain a useful panoramic radiograph. Therefore, panoramic radiographs are harder to assess than intraoral radiographs, and assessing panoramic radiographs requires extra training. The intra- and inter-observer agreement for panoramics is lower [55]. However, intraoral radiographs were not included in the original Prospective Population Study of Women in Gothenburg.

Moreover, it might be that the situation is different from the viewpoint of texture analysis. In a previous study, the texture analysis methods were applied on panoramic radiographs as well as on intraoral radiographs [10]. The measurements were used to predict total hip BMD and spinal BMD. It was found that panoramic radiographs on average contributed more to the predictions than intraoral radiographs of the mandible and maxilla. A related study used the texture analysis methods to predict osteoporosis [30]. After age, the second most important predictor originated from the panoramic radiographs whereas the next most important predictor originated from intraoral radiographs of the mandible. This demonstrates that panoramic radiographs may be more important than intraoral radiographs when it comes to predicting BMD and or osteoporosis.

It is shown that the trabecular pattern on panoramic radiographs contains important clues for predicting postcranial fractures. These clues are picked up by the human observer and to a lesser extent by the texture analysis procedures even though image features like width and area of the "trabeculae" and "marrow spaces" had been designed to quantify the coarseness of the trabecular pattern. This may be due to the knowledge that an experienced dentists has of the human anatomy.

\section{Conclusion}

Conclusively, it can be said that the observer score of the trabecular pattern is a sophisticated evaluation not yet achieved by machine analysis. The present study demonstrates that the radiographic trabecular pattern contains information for a state of the art prediction of future fractures, at least in postmenopausal women. Considering the social burden of fractures and the low costs involved with panoramic radiographs, further study of the radiographic trabecular pattern is justified.

\section{Compliance with ethical standards}

Conflict of interest The authors declare that they have no conflict of interest.

Funding This research did not receive any specific grant from funding agencies in the public, commercial, or not-for-profit sectors.

Ethical approval For retrospective studies, formal consent is not required.

Informed consent Participants gave their informed consent in accordance with the Helsinki Declaration.

Open Access This article is distributed under the terms of the Creative Commons Attribution 4.0 International License (http:// creativecommons.org/licenses/by/4.0/), which permits unrestricted use, distribution, and reproduction in any medium, provided you give appropriate credit to the original author(s) and the source, provide a link to the Creative Commons license, and indicate if changes were made.

\section{References}

1. Cummings SR, Melton LJ (2002) Epidemiology and outcomes of osteoporotic fractures. Lancet 359:1761-1767. doi:10.1016/s01406736(02)08657-9

2. Surgeon_General (2004) Bone Health and Osteoporosis: A Report of the Surgeon General. Book title. Department of Health and Human Services, Office of the Surgeon General, Rockville, MD: U.S 
3. Hernlund E, Svedbom A, Ivergard M, Compston J, Cooper C, Stenmark J, McCloskey EV, Jonsson B, Kanis JA (2013) Osteoporosis in the European Union: medical management, epidemiology and economic burden. A report prepared in collaboration with the international osteoporosis Foundation (IOF) and the European Federation of Pharmaceutical Industry Associations (EFPIA). Arch Osteoporos 8:136. doi:10.1007/s11657-013-0136-1

4. Kanis JA, Melton LJ 3rd, Christiansen C, Johnston CC, Khaltaev N (1994) The diagnosis of osteoporosis. J Bone Miner Res 9:11371141. doi: $10.1002 / \mathrm{jbmr} .5650090802$

5. Kanis JA (2002) Diagnosis of osteoporosis and assessment of fracture risk. Lancet 359:1929-1936. doi:10.1016/s0140-6736(02) 08761-5

6. Siris ES, Chen YT, Abbott TA, Barrett-Connor E, Miller PD, Wehren LE, Berger ML (2004) Bone mineral density thresholds for pharmacological intervention to prevent fractures. Arch Intern Med 164:1108-1112. doi:10.1001/archinte.164.10.1108

7. Pasco JA, Seeman E, Henry MJ, Merriman EN, Nicholson GC, Kotowicz MA (2006) The population burden of fractures originates in women with osteopenia, not osteoporosis. Osteoporos Int 17: 1404-1409. doi:10.1007/s00198-006-0135-9

8. Nguyen ND, Eisman JA, Center JR, Nguyen TV (2007) Risk factors for fracture in nonosteoporotic men and women. J Clin Endocrinol Metab 92:955-962. doi:10.1210/jc.2006-1476

9. Kanis JA, Johnell O, Oden A, Johansson H, McCloskey E (2008) FRAX and the assessment of fracture probability in men and women from the UK. Osteoporos Int 19:385-397. doi:10.1007/s00198007-0543-5

10. Geraets WG, Verheij JG, van der Stelt PF, Horner K, Lindh C, Nicopoulou-Karayianni K, Jacobs R, Harrison EJ, Adams JE, Devlin H (2007) Prediction of bone mineral density with dental radiographs. Bone 40:1217-1221. doi:10.1016/j.bone.2007.01.009

11. Warriner AH, Patkar NM, Yun H, Delzell E (2011) Minor, major, low-trauma, and high-trauma fractures: what are the subsequent fracture risks and how do they vary? Curr Osteoporos Rep 9:122128. doi:10.1007/s11914-011-0064-1

12. Fawcett T (2006) An introduction to ROC analysis. Pattern Recogn Lett 27:861-874. doi:10.1016/j.patrec.2005.10.010

13. National Clinical Guideline C (2012) National Institute for Health and clinical excellence: guidance. Royal College of Physicians (UK) National Clinical Guideline Centre, London

14. Tremollieres FA, Pouilles JM, Drewniak N, Laparra J, Ribot CA, Dargent-Molina P (2010) Fracture risk prediction using BMD and clinical risk factors in early postmenopausal women: sensitivity of the WHO FRAX tool. J Bone Miner Res 25:1002-1009. doi:10. 1002/jbmr. 12

15. Donaldson MG, Cawthon PM, Schousboe JT, Ensrud KE, Lui LY, Cauley JA, Hillier TA, Taylor BC, Hochberg MC, Bauer DC, Cummings SR, Study of Osteoporotic F (2011) Novel methods to evaluate fracture risk models. J Bone Miner Res 26:1767-1773. doi:10.1002/jbmr.371

16. Donaldson MG, Palermo L, Schousboe JT, Ensrud KE, Hochberg MC, Cummings SR (2009) FRAX and risk of vertebral fractures: the fracture intervention trial. J Bone Miner Res 24:1793-1799. doi: 10.1359/jbmr.090511

17. Rubin KH, Abrahamsen B, Friis-Holmberg T, Hjelmborg JV, Bech M, Hermann AP, Barkmann R, Gluer CC, Brixen K (2013) Comparison of different screening tools (FRAX(R), OST, ORAI, OSIRIS, SCORE and age alone) to identify women with increased risk of fracture. A population-based prospective study. Bone 56:1622. doi:10.1016/j.bone.2013.05.002

18. Ensrud KE, Lui LY, Taylor BC, Schousboe JT, Donaldson MG, Fink HA, Cauley JA, Hillier TA, Browner WS, Cummings SR (2009) A comparison of prediction models for fractures in older women: is more better? Arch Intern Med 169:2087-2094. doi:10. 1001/archinternmed.2009.404
19. Black DM, Steinbuch M, Palermo L, Dargent-Molina P, Lindsay R, Hoseyni MS, Johnell O (2001) An assessment tool for predicting fracture risk in postmenopausal women. Osteoporos Int 12:519528. doi:10.1007/s001980170072

20. Miller PD, Siris ES, Barrett-Connor E, Faulkner KG, Wehren LE, Abbott TA, Chen YT, Berger ML, Santora AC, Sherwood LM (2002) Prediction of fracture risk in postmenopausal white women with peripheral bone densitometry: evidence from the National Osteoporosis Risk Assessment. J Bone Miner Res 17:2222-2230. doi:10.1359/jbmr.2002.17.12.2222

21. Sandhu SK, Nguyen ND, Center JR, Pocock NA, Eisman JA, Nguyen TV (2010) Prognosis of fracture: evaluation of predictive accuracy of the FRAX algorithm and Garvan nomogram. Osteoporos Int 21:863-871. doi:10.1007/s00198-009-1026-7

22. Ahmed LA, Nguyen ND, Bjornerem A, Joakimsen RM, Jorgensen L, Stormer J, Bliuc D, Center JR, Eisman JA, Nguyen TV, Emaus N (2014) External validation of the Garvan nomograms for predicting absolute fracture risk: the Tromso study. PLoS One 9:e107695. doi: 10.1371/journal.pone.0107695

23. Crandall CJ, Larson JC, Watts NB, Gourlay ML, Donaldson MG, LaCroix A, Cauley JA, Wactawski-Wende J, Gass ML, Robbins JA, Ensrud KE (2014) Comparison of fracture risk prediction by the US preventive services task force strategy and two alternative strategies in women 50-64 years old in the Women's Health Initiative. J Clin Endocrinol Metab 99:4514-4522. doi:10.1210/jc. 2014-2332

24. Devlin H, Allen PD, Graham J, Jacobs R, Karayianni K, Lindh C, van der Stelt PF, Harrison E, Adams JE, Pavitt S, Horner K (2007) Automated osteoporosis risk assessment by dentists: a new pathway to diagnosis. Bone 40:835-842. doi:10.1016/j.bone.2006.10.024

25. Geraets WG, Verheij JG, van der Stelt PF, Horner K, Lindh C, Nicopoulou-Karayianni K, Jacobs R, Marjanovic EJ, Adams JE, Devlin H (2008) Selecting regions of interest on intraoral radiographs for the prediction of bone mineral density. Dento maxillo facial radiology 37:375-379. doi:10.1259/dmfr/29966973

26. Jonasson G, Jonasson L, Kiliaridis S (2007) Skeletal bone mineral density in relation to thickness, bone mass, and structure of the mandibular alveolar process in dentate men and women. Eur $\mathbf{J}$ Oral Sci 115:117-123. doi:10.1111/j.1600-0722.2007.00438.x

27. Jonasson G (2009) Bone mass and trabecular pattern in the mandible as an indicator of skeletal osteopenia: a 10-year follow-up study. Oral Surg Oral Med Oral Pathol Oral Radio Lendodontics 108:284 291. doi:10.1016/j.tripleo.2009.01.014

28. Lee BD, White SC (2005) Age and trabecular features of alveolar bone associated with osteoporosis. Oral Surg Oral Med Oral Pathol Oral Radiol Endodontics 100:92-98. doi:10.1016/j.tripleo.2004.11. 020

29. Lindh C, Horner K, Jonasson G, Olsson P, Rohlin M, Jacobs R, Karayianni K, van der Stelt P, Adams J, Marjanovic E, Pavitt S, Devlin H (2008) The use of visual assessment of dental radiographs for identifying women at risk of having osteoporosis: the OSTEODENT project. Oral Surg Oral Med Oral Pathol Oral Radiol Endodontics 106:285-293. doi:10.1016/j.tripleo.2007.09. 008

30. Verheij JG, Geraets WG, van der Stelt PF, Horner K, Lindh C, Nicopoulou-Karayianni K, Jacobs R, Marjanovic EJ, Adams JE, Devlin H (2009) Prediction of osteoporosis with dental radiographs and age. Dento maxillo facial radiology 38:431-437. doi:10.1259/ $\mathrm{dmfr} / 55502190$

31. White SC, Taguchi A, Kao D, Wu S, Service SK, Yoon D, Suei Y, Nakamoto T, Tanimoto K (2005) Clinical and panoramic predictors of femur bone mineral density. Osteoporos Int 16:339-346. doi:10. 1007/s00198-004-1692-4

32. Hassani-Nejad A, Ahlqwist M, Hakeberg M, Jonasson G (2013) Mandibular trabecular bone as fracture indicator in 80-year-old men and women. Eur J Oral Sci 121:525-531. doi:10.1111/eos.12087 
33. Jonasson G, Alstad T, Vahedi F, Bosaeus I, Lissner L, Hakeberg M (2009) Trabecular pattern in the mandible as bone fracture predictor. Oral Surg Oral Med Oral Pathol Oral Radiol Endodontics 108: e42-e51. doi:10.1016/j.tripleo.2009.05.018

34. Jonasson $\mathrm{G}$, Sundh V, Ahlqwist $\mathrm{M}$, Hakeberg M, Bjorkelund C, Lissner L (2011) A prospective study of mandibular trabecular bone to predict fracture incidence in women: a low-cost screening tool in the dental clinic. Bone 49:873-879. doi:10.1016/j.bone.2011.06. 036

35. Jonasson G, Sundh V, Hakeberg M, Hassani-Nejad A, Lissner L, Ahlqwist M (2013) Mandibular bone changes in 24 years and skeletal fracture prediction. Clin Oral Investig 17:565-572. doi:10. 1007/s00784-012-0745-x

36. White SC, Atchison KA, Gornbein JA, Nattiv A, Paganini-Hill A, Service SK, Yoon DC (2005) Change in mandibular trabecular pattern and hip fracture rate in elderly women. Dento maxillo facial radiology 34:168-174. doi:10.1259/dmfr/32120028

37. Lindh C, Petersson A, Rohlin M (1996) Assessment of the trabecular pattern before endosseous implant treatment: diagnostic outcome of periapical radiography in the mandible. Oral Surg Oral Med Oral Pathol Oral Radiol Endodontics 82:335-343

38. Korstjens CM, Geraets WG, van Ginkel FC, Prahl-Andersen B, van der Stelt PF, Burger EH (1994) An analysis of the orientation of the radiographic trabecular pattern in the distal radius of children. Growth Dev Aging 58:211-221

39. Geraets WG, Van der Stelt PF, Lips P, Van Ginkel FC (1998) The radiographic trabecular pattern of hips in patients with hip fractures and in elderly control subjects. Bone 22:165-173

40. Geraets WG, Van der Stelt PF, Elders PJ (1993) The radiographic trabecular bone pattern during menopause. Bone 14:859-864

41. Geraets WG, Lindh C, Verheij H (2012) Sparseness of the trabecular pattern on dental radiographs: visual assessment compared with semi-automated measurements. Br J Radiol 85:e455-e460. doi:10.1259/bjr/32962542

42. White SC, Rudolph DJ (1999) Alterations of the trabecular pattern of the jaws in patients with osteoporosis. Oral Surg Oral Med Oral Pathol Oral Radiol Endodontics 88:628-635

43. White SC, Cohen JM, Mourshed FA (2000) Digital analysis of trabecular pattern in jaws of patients with sickle cell anemia. Dento maxillo facial radiology 29:119-124. doi:10.1038/sj/dmfr/ 4600516

44. Geraets WGM, Van der Stelt PF (1991) Analysis of the radiographic trabecular pattern. Pattern Recogn Lett 12:575-581. doi:10.1016/ 0167-8655(91)90168-L
45. Bengtsson C, Ahlqwist M, Andersson K, Bjorkelund C, Lissner L, Soderstrom M (1997) The prospective population study of women in Gothenburg, Sweden, 1968-69 to 1992-93. A 24-year follow-up study with special reference to participation, representativeness, and mortality. Scand J Prim Health Care 15:214-219

46. Sundh V, Hange D, Ahlqwist M, Hakeberg M, Lissner L, Jonasson $\mathrm{G}$ (2017) FRAX and mandibular sparse trabeculation as fracture predictors: a longitudinal study from 1980 to 2002. Eur J Oral Sci 125:135-140. doi:10.1111/eos.12341

47. Geraets WG, Verheij JG, van der Stelt PF, Horner K, Lindh C, Nicopoulou-Karayianni K, Jacobs R, Devlin H (2007) Osteoporosis and the general dental practitioner: reliability of some digital dental radiological measures. Community Dent Oral Epidemiol 35:465-471. doi:10.1111/j.1600-0528.2006.00357.x

48. Geraets WG (1998) Comparison of two methods for measuring orientation. Bone 23:383-388

49. Zhang ZM, Li ZC, Jiang LS, Jiang SD, Dai LY (2010) Micro-CT and mechanical evaluation of subchondral trabecular bone structure between postmenopausal women with osteoarthritis and osteoporosis. Osteoporos Int 21:1383-1390. doi:10.1007/s00198-009-10712

50. Kanis JA, Johnell O, Oden A, Sembo I, Redlund-Johnell I, Dawson A, De Laet C, Jonsson B (2000) Long-term risk of osteoporotic fracture in Malmo. Osteoporos Int 11:669-674

51. Leslie WD, Lix LM (2014) Comparison between various fracture risk assessment tools. Osteoporos Int 25:1-21. doi:10.1007/ s00198-013-2409-3

52. Calciolari E, Donos N, Park JC, Petrie A, Mardas N (2015) Panoramic measures for oral bone mass in detecting osteoporosis: a systematic review and meta-analysis. J Dent Res $94: 17 \mathrm{~s}-27 \mathrm{~s}$. doi: 10.1177/0022034514554949

53. Kersh ME, Pandy MG, Bui QM, Jones AC, Arns CH, Knackstedt MA, Seeman E, Zebaze RM (2013) The heterogeneity in femoral neck structure and strength. J Bone Miner Res 28:1022-1028. doi: 10.1002/jbmr.1827

54. Zebaze RM, Ghasem-Zadeh A, Bohte A, Iuliano-Burns S, Mirams M, Price RI, Mackie EJ, Seeman E (2010) Intracortical remodelling and porosity in the distal radius and post-mortem femurs of women: a cross-sectional study. Lancet (London, England) 375:1729-1736. doi:10.1016/s0140-6736(10)60320-0

55. Pham D, Jonasson G, Kiliaridis S (2010) Assessment of trabecular pattern on periapical and panoramic radiographs: a pilot study. Acta Odontol Scand 68:91-97. doi:10.3109/00016350903468235 